\title{
Registro Nacional de la hemorragia subaracnoídea en Uruguay: descripción de los primeros 36 casos
}

\author{
National database of spontaneous aneurysmal subarachnoid \\ hemorrhage in Uruguay. Description of the first 36 cases
}

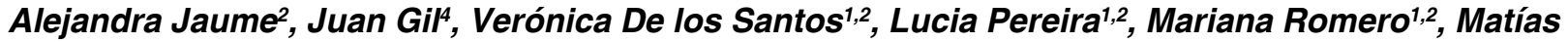 \\ Negrotto $^{3}$, J. Pedro Macadam ${ }^{1,2}$, Fernando Martínez', Edgardo Spagnuolo ${ }^{2}$ \\ ${ }^{1}$ Cátedra de Neurocirugía, Hospital de Clínicas de Montevideo, Universidad de la República. Uruguay. \\ ${ }^{2}$ Servicio de Neurocirugía, Hospital Maciel, ASSE, Uruguay. \\ ${ }^{3}$ Cátedra de Imagenología Hospital de Clínicas, Montevideo. Universidad de la República. Uruguay. \\ ${ }^{4}$ Departamento de Métodos Cuantitativos, Facultad de Medicina, Montevideo. Universidad de la República. Uruguay.
}

\section{Resumen}

Introducción: La HSA constituye del 3 al 5\% de todos los ataques cerebro vasculares en la población general. Es una enfermedad de alto impacto en la sociedad, por su elevada morbimortalidad. Se han realizado múltiples estudios retrospectivos, pero no hay ningún estudio prospectivo nacional actualizado de dicha patología en Uruguay. Aquí se publican datos preliminares de un trabajo anual con el objetivo de conocer las características demográficas, epidemiológicas, así como las características topográficas y morfo-estructurales de los aneurismas, y la evolución de los pacientes portadores de esta patología. Materiales y Métodos: Se esta realizando un trabajo multicéntrico de una cohorte longitudinal prospectivo de todos los pacientes que se presentan con HSA en el Uruguay en un período de un año. En esta presentación preliminar se incluyen para un primer análisis todos los pacientes entre el 1 de noviembre de 2019 y el 31 de enero de 2020. Se incluyeron todos los pacientes mayores de 18 años que consultaron y se les diagnosticó una HSA espontánea. Una vez hecho el diagnóstico de HSA, dependiendo de la gravedad del paciente se realizó un estudio vascular (angio-TC o arteriografía) para valorar la presencia o no de aneurisma, y la topografía del mismo para decidir el tratamiento. Se analizaron las siguientes variables: edad, sexo, procedencia geográfica, antecedentes, motivo de consulta, escala de coma de Glasgow (GCS), escala de World Federation of Neurological Surgeons (WFNS), escala Hunt y Hess, escala de Fisher, consultas previas al ingreso, tratamiento indicado, complicación vinculada a la HSA, complicación vinculada al tratamiento indicado, y escala de rankin modificado (mRS) al mes. Se creó una máscara de captura, y el análisis se procesó utilizando el software SPSS versión 22. La investigación fue aprobada por todos los Comité de Ética de los diferentes centros que participaron del estudio. Resultados: De un total de 36 pacientes, la edad media fue de 55,2 años, con mayor frecuencia en el sexo femenino. En el $83,3 \%$ de los pacientes el síntoma que predominó fue la cefalea y en la mitad de los casos estuvo asociada a vómitos. El trastorno de conciencia se presentó en $55 \%$ de los casos. Hubo $25 \%$ de error diagnóstico. El estudio diagnóstico de aneurisma más frecuente usado fue la angio TC en el $80 \%$ de los casos, y la arteriografía se realizó en el $41 \%$. En el $63 \%$ de los casos se realizó cirugía abierta, y en el $14 \%$ se realizó tratamiento endovascular. Los aneurismas más frecuentes fueron el comunicante anterior, el silviano y el comunicante posterior. El $80 \%$ presentó complicaciones vinculadas a su HSA. A los 30 días del ictus 11 pacientes $(30 \%)$ fallecieron (mRS 6), en la gran mayoría de los casos la causa fue neurológica. Debemos destacar que la mayoría de los pacientes que fallecieron ingresaron con un mal estado neurológico. Conclusiones: Este trabajo presenta las primeras cifras sobre las características epidemiológicas de la Hemorragia subaracnoidea en el Uruguay.

Palabras clave: Hemorragia meníngea, stroke, epidemiología, tratamiento de aneurismas, pronóstico.

\section{Correspondencia a:}

Dra. Alejandra Jaume

Neurocirujana, Hospital Maciel. Montevideo, Uruguay.

Camino Carrasco 6093. CP: 11500. Montevideo, Uruguay.

(00598) 26010891-(00598) 99273939

ale.jaume@ hotmail.com 


\begin{abstract}
Introduction: The SAH constitutes from 3 to $5 \%$ of all strokes in general population. It is a disease of high impact on society, because of its high morbidity and mortality. Multiple retrospective studies have been carried out, but there is no updated national prospective study of this pathology in Uruguay. Preliminary data from an annual study are published here with the aim of knowing the demographic, epidemiological characteristics, as well as the topographic and morpho-structural characteristics of aneurysms, and the evolution of patients with this pathology. Materials and Methods: A multicenter study of a prospective longitudinal cohort of all patients who presented with SAH in Uruguay in a period of one year is carried out, including in this first analysis all patients between November 1, 2019 and January 31, 2020. All patients older than 18 years who consulted were included. They were diagnosed with spontaneous $\mathrm{SAH}$. Once the diagnosis of SAH was made, depending on the severity of the patient, a vascular study was performed (angio-CT or arteriography) to assess the presence or absence of aneurysm, and the topography of it to decide the treatment. The following variables were analyzed: age, sex, geographical origin, history, reason for consultation, Glasgow Coma Scale (GCS), World Federation of Neurological Surgeons (WFNS) scale, Hunt and Hess scale, Fisher scale, previous consultations. at admission, indicated treatment, complication related to $\mathrm{SAH}$, complication related to indicated treatment, and modified rankin scale (mRS) at month. A capture mask was created, and the analysis was processed using SPSS version 22 software. The research was approved by all the Ethics Committee of the different centers that participated in the study. Results: out of a total of 36 patients, the average age was 55,2 years, more frequently in females. In $83,3 \%$ of the patients the predominant symptom was headache and in half of the cases it was associated with vomiting. Loss of consciousness occurred in $55 \%$ of cases. There was a $25 \%$ diagnostic error. The diagnostic study of the most frequent aneurysm used was CT angiography in $80 \%$ of cases, and arteriography was performed in $41 \%$. Open surgery was performed in $63 \%$ of cases, and endovascular treatment in $14 \%$ of cases. The most frequent aneurysms were anterior communicant, sylvian and posterior communicating. $80 \%$ presented complications related to their SAH. 30 days after stroke, 11 patients (30\%) died (mRS 6), in the great majority of cases the cause was neurological. We must emphasize that the majority of patients who died were admitted with a poor neurological status. Conclusions: This paper presents the first figures on the annual incidence and epidemiological characteristics of Subarachnoid Hemorrhage in Uruguay.
\end{abstract}

Keywords: meningeal hemorrhage, stroke, epidemiology, treatment of aneurysms, weather forecast.

\section{Introducción}

El ataque cerebro-vascular (ACV) constituye la primera causa de morbilidad, y la tercera causa de mortalidad en el mundo ${ }^{1}$. Es una de las patologías más importantes actualmente, siendo la prevención un pilar fundamental, dado que una vez que se produce, las consecuencias pueden ser desvastadoras $^{2}$. El ACV hemorrágico tiene una frecuencia entre $10-30 \%$ de todos los ACV, pero su morbimortalidad es mayor con respecto al ACV isquemico ${ }^{3}$. La hemorragia subaracnoidea (HSA), representa solo el 3-5\% de todos los stroke ${ }^{4}$, siendo una patología de alto impacto en la sociedad, por su elevada morbi-mortalidad, con una mortalidad global aproximadamente del $40 \% 5$.

El rango de prevalencia de la HSA en la población general es de $0,2 \%$ al $9,9 \%{ }^{6}$.

La incidencia de dicha patología varía según su población etárea, desde 6/100.000 habitantes por año en países como China, hasta 22/100.000 habitantes por año, en países como Finlandia y Japón ${ }^{7}$. Dicha incidencia depende de factores genéticos predisponentes, así como de características de la población etárea y del país en estudio ${ }^{8}$.

La clínica de presentación es variada, por lo cual es importante la sospecha de esta patología, para lograr un diagnóstico oportuno. La HSA es más frecuente entre la cuarta y sexta década de edad, y es más frecuente en mujeres ${ }^{9}$.

El 80 a $85 \%$ de las HSA se deben a rotura de un aneuris- $\mathrm{ma}^{10}$. Los aneurismas intracraneanos pueden ser clasificados de diferentes maneras: según su etiología, localización, tamaño, o morfología. Los aneurismas saculares son los más frecuentes, y pueden tener diferentes etiologías, siendo la mayoría de causa espontánea, producidos por stress hemodinámico ${ }^{11}$.

En los últimos años en Uruguay se han realizado algunos estudios retrospectivos, que apuntaron a describir la evolución de los pacientes con HSA, pero no existe ningún estudio prospectivo a nivel nacional que haya permitido estimar la incidencia de dicha patología en nuestro país.

Acorde a esto, surge la necesidad de realizar un proyecto nacional, que permita estimar la incidencia de la HSAea en el país, estudio que se está llevando a cabo en este momento.

El objetivo de este trabajo es presentar los resultados preliminares de este estudio multicéntrico, describiendo los primeros 36 casos incluidos en cuanto a sus características demográficas, epidemiológicas, topográficas y morfo-estructurales de los aneurismas, asi como de su evolución inmediata.

\section{Materiales y Métodos}

Se trata de un estudio prospectivo y multicéntrico, incluyendo a todos los pacientes que presentan HSA en el Uruguay entre el 1 de noviembre de 2019 y el 31 de octubre de 2020. A los efectos de esta publicación preliminar, se con- 
sideró como fecha de fin de inclusión el 31 de enero de 2020 y como fecha de fin de seguimiento el 1 de marzo de 2020.

Fueron incluidos todos los pacientes mayores de 18 años que consultaron y se les diagnosticó una HSA espontánea en el período del trimestre mencionado anteriormente. Los pacientes de edad pedíatrica no fueron incluidos en el trabajo dado que la etiología y fisiopatología de la HSA en dicha edad tiene connotaciones y terapéuticas diferentes. Se registraron los datos en el momento de la atención primaria, e independientemente del estado clínico a todos los pacientes se le realizó Tomografía axial computada (TAC), punción lumbar, o Resonancia Nuclear Magnética, confirmando la presencia de HSA. Una vez hecho el diagnóstico de HSA, dependiendo de la gravedad del paciente se realizó un estudio vascular (angio-TC o arteriografía) para valorar la presencia o no de aneurisma, y la topografía del mismo para decidir el tratamiento. En los casos de mayor gravedad que no se llegó a realizar un estudio vascular, dichos pacientes fueron igualmente incluidos para determinados datos epidemiológicos, quedando fuera del resto de los datos extraídos para la parte específica de las variables en relación al aneurisma. Se excluyeron aquellos pacientes cuyo estudio vascular fue negativo para aneurisma, y aneurismas traumáticos o micóticos.

Los datos fueron recolectados mediante un formulario único, completado siempre por el mismo integrante del equipo. Previo a recabar estos datos, se le solicitó a cada paciente o su representante (si el paciente no tenía capacidad para consentir), su consentimiento a participar del estudio, luego de informarle sobre las implicancias de su participación.

Se analizaron las siguientes variables: edad, sexo, procedencia geográfica, antecedentes personales del paciente, centro asistencial de ingreso, motivo de consulta, fecha y hora de inicio de los síntomas, fecha y hora de ingreso al centro asistencial, escala de coma de Glasgow (GCS), escala de World Federation of Neurological Surgeons (WFNS), escala Hunt y Hess, escala de Fisher, fecha y hora del tratamiento indicado, estudio(s) vascular realizado(s), consultas previas al ingreso, topografía del aneurisma que sangró, presencia de otros aneurismas, fecha y hora de inicio del tratamiento realizado, centro asistencial donde se realizó el tratamiento, complicación vinculada a la HSA, complicación vinculada al tratamiento indicado, y escala de rankin modificado (mRS) al mes.

Respecto al análisis estadístico de los datos, las características de los pacientes fueron resumidas por porcentajes en el caso de variables cualitativas y por medias y/o medianas en el caso de variables cuantitativas.

Toda la información recabada en este estudio es confidencial y fue manejada según las normas éticas para estudios de investigación epidemiológica. La identidad de los pacientes fue manejada únicamente por los médicos intervinientes en el proceso asistencial. Una base de datos informatizada fue creada con el solo fin de procesar estadísticamente la información recabada, manteniendo en la misma, los datos de los pacientes de forma no-identificada, a través de un código asignado a cada paciente, únicamente conocido por parte de los investigadores del estudio.

Los centros participantes del estudio fueron aquellos que diagnostican y tratan esta patología en el Uruguay: Servicios clínicos dependientes de la Facultad de Medicina de la
UdelaR, hospitales de los servicios de salud pública (ASSE), centros asistenciales privados, y los 3 centros que realizan neuro-intervencionismo en el país.

La investigación fue aprobada previamente por todos los Comité de Ética institucionales, así como también por la Comisión Nacional de Ética en Investigación (CNEI), por tratarse de un estudio multicéntrico.

Además cuenta con el soporte y aval de la Sociedad Uruguaya de Neurocirugía (SUNC).

\section{Resultados}

En el período de estudio descrito en este trabajo (primer trimestre del estudio original) fue incluido un total de 36 casos.

Uruguay esta formado por 19 departamentos, siendo Montevideo la capital y departamento más poblado de nuestro país. En cuanto a la procedencia, el porcentaje encontrado fue similar tomando en cuenta Montevideo con respecto al interior del país, $52,8 \%$ y $47,2 \%$ respectivamente.

La media de edad (en años) fue de 55,2 (IC95\%: 51,1$59,4)$, siendo la mayor proporción (70\%) entre los $40-70$ años.

Las características socio-demográficas y antecedentes personales relevantes de los pacientes incluidos se resumen en la Tabla 1.

En el 83,3\% de los pacientes el síntoma que predominó fue la cefalea y en la mitad de los casos estuvo asociada a vómitos. El trastorno de conciencia se presentó en el 55\% de los casos: el $14 \%$ de estos fue transitoria, $11 \%$ coma brusco y $30 \%$ con una depresión de conciencia progresiva con puntuación en la escala de coma de GCS intermedio entre 9 y 13. La presencia de crisis epiléptica como síntoma ocurrió en el $13,9 \%$. El déficit neurológico fue poco frecuente $2,8 \%$ y la alteración del tercer par craneano en 5,6\% de los casos, siendo causado por fenómenos locales como irritación y/o compresión del mismo, vinculados a un aneurisma comunicante posterior. No se incluyeron en este ítem los pacientes con anisocoria por HEC.

En el momento del diagnóstico de HSA se utilizarón las clasificaciones aceptadas internacionalmente para esta patología como son la: World Federation Neurosurgical Score (WFNS) (Tabla 2), la clasificación de Hunt y Hess $(\mathrm{HyH})(\mathrm{Ta}-$

Tabla 1. Características de los pacientes incluidos

(\%) de pacientes ( $n=36)$

\begin{tabular}{lc|} 
Sexo femenino & $61 \%$ \\
Raza caucásica & $97 \%$ \\
Nacionalidad uruguaya & $94,4 \%$ \\
Salud pública & $53 \%$ \\
Antecedente de HTA & $64 \%$ \\
Antecedente de fumador & $50 \%$ \\
Antecedente personal y/o familiar de & $5,6 \%$ \\
aneurisma & \\
Consultas previo al ingreso & $25 \%$ \\
\hline
\end{tabular}


bla 3), y la escala de Fisher modificada (Tabla 4), obteniendo los resultados que se detallan en las tablas.

En el $36 \%$ de los casos en dicha tomografía inicial se evidenció además la presencia de hidrocefalia (HCF).

En el $80 \%$ de los casos el estudio diagnóstico de aneurisma fue con angio TC. La arteriografía se realizó en el $41 \%$. De estas $16 \%$ fueron con fines diagnósticos porque la angioTC no fue concluyente y el resto con fines terapéuticos. En algunos casos no se llegó a realizar ningún estudio vascular por mala condición neurológica del paciente al ingreso.

Se realizó tratamiento quirúrgico en el $63 \%$, tratamiento endovascular en el $14 \%$, y en el resto no se realizó ningún tipo de tratamiento por la situación neurológica grave del paciente. Solo un caso se indicó tratamiento endovascular y se termino clipando por cirugía abierta por dificultad en el acceso al aneurisma. Se destaca, que dentro de los pacientes que se operaron, la decisión es tomada en la mayoría de los casos solo por el neurocirujano actuante, en cambio en los casos de embolización fue más frecuente la decisión en conjunto del neurocirujano y equipo endovascular. En 14\% de los casos el hallazgo imagenológico de lesión ocupante de espacio, fue determinante para la elección quirúrgica del tratamiento

\section{Tabla 2. Escala de WFNS}

\begin{tabular}{|llc|}
\hline \multicolumn{3}{|c|}{ Tabla 2. Escala de WFNS } \\
\hline & & $\begin{array}{c}\text { (\%) de pacientes } \\
\text { (n = 36) }\end{array}$ \\
\hline 1 & GSC 15, sin hemiparesia & $30 \%$ \\
2 & GSC 13-14 sin hemiparesia & $9 \%$ \\
3 & GSC 13-14 con hemiparesia & $6 \%$ \\
4 & GSC 7-12 con o sin hemiparesia & $19 \%$ \\
5 & GSC 3-6 con o sin hemiparesia & $36 \%$ \\
\hline
\end{tabular}

del aneurisma.

Tomando en cuenta los aneurismas rotos, se evidenció que los 3 más frecuentes fueron el comunicante anterior $30 \%$, el silviano $28 \%$ y luego el comunicante posterior en $22 \%$. Estos en su conjunto suman el $80 \%$ del total de aneurismas.

En cuanto a las caractéristicas del aneurisma en $78 \%$ fueron de tipo sacular, y un 53\% tenía el cuello chico en relación al cuello.

La presencia de aneurismas múltiples, corresponden a un $22 \%$ del total de pacientes. Lo más frecuente es que tenga un solo aneurisma testigo, siendo el $62 \%$ de los casos, y en el resto de los casos se presentaron con dos aneurismas testigos.

Las complicaciones de la HSA son de alta frecuencia, en este trabajo observamos $80 \%$ de complicaciones vinculadas a la HSA. Dentro de estas lo menos frecuente fue el resangrado que se evidenció en tan solo 2 pacientes, y el hematoma parenquimatoso que se presento en $16 \%$.

El 44\% presentó vasoespasmo, sabemos que este diagnóstico es sobre todo arteriográfico, pero dado que hay muy baja cantidad de pacientes que se realizó arteriografía en este período, tomamos en cuenta los pacientes con isquemia en diferido al tratamiento del aneurisma con características imagenológicas de infarto por vasoespasmo.

La hidrocefalia es la complicación más frecuente $64 \%$, en casi todos los casos es aguda, representada por una dilatación discreta del sistema ventricular en la mayoría de los casos. De este porcentaje de pacientes con HCF, en el $87 \%$ se realizó algún tratamiento específico para esta complicación, ya sea con drenaje lumbar continuo, derivación ventricular externa, tercer-ventriculostomía, y/o derivación ventricular interna. Solo en el $13 \%$ no se hizo nada dada la gravedad del paciente.

En cuanto a las complicaciones vinculadas al tratamiento

Tabla 3. Escala de Hunt y Hess

(\%) de pacientes $(n=36)$

\section{Asintomático o mínima cefalea con leve rigidez de nuca}

2 Cefalea severa-moderada con rigidez de nuca, sin déficit focal neurológico, salvo alteración de par craneano

3 Somnolencia, confusión o déficit focal leve

4 Estupor, déficit motor moderado-severo, signos precoces de descerebración o alteraciones vegetativas

5 Coma profundo, rigidez de descerebración, aspecto moribundo

$9 \%$
$28 \%$
$11 \%$
$22 \%$
$30 \%$

\section{Tabla 4. Escala de Fisher modificada}

\begin{tabular}{|c|c|c|}
\hline Fisher 1 & Sin evidencia de sangrado en cisternas y ventrículos & $0 \%$ \\
\hline Fisher 2 & Sangre difusa fina, con una capa de menos de $1 \mathrm{~mm}$ en la cisternas & $14 \%$ \\
\hline Fisher 3 & Coagulo grueso cisternal mayor a $1 \mathrm{~mm}$, hemocisterna & $39 \%$ \\
\hline Fisher 4 & Hematoma intraparenquimatoso, hemorragia intraventricular & $0 \%$ \\
\hline Fisher $2+4$ & & $6 \%$ \\
\hline Fisher $3+4$ & & $41 \%$ \\
\hline
\end{tabular}




\section{Tabla 5. Escala de mRS}

0 Asintomático

1 Discapacidad no significativa a pesar de los síntomas, capaz de llevar a cabo todas las tareas y actividades habituales

2 Discapacidad leve, incapaz de llevar a cabo todas sus actividades anteriores, pero capaz de cuidar de sus propios asuntos sin ayuda

3 Discapacidad moderada, requiere alguna asistencia, pero es capaz de andar sin ayuda

4 Discapacidad moderadamente severa, incapaz de andar y de atender satisfactoriamente sus necesidades corporales sin ayuda

5 Discapacidad severa, confinamiento en la cama, incontinencia y requerimiento de cuidados y atenciones constantes

6 Muerte

realizado, hubieron 9 casos (25\%), siendo $6(66 \%)$ casos de cirugía abierta, y 3 (34\%) del tratamiento endovascular.

Dentro de las complicaciones se incluyó: la rotura intraprocedimiento del aneurisma, el clipado transitorio largo, la disección de vaso, isquemia grande relacionada al sitio tratado, y/o isquemia pequeña cerca o lejos de la topografía tratada. De todas estas la más frecuente fue la isquemia pequeña en $44 \%$ de los casos.

De los pacientes que recibieron tratamiento (cirugía o embolización), $72 \%$ fue en las primeras 24 horas posterior al ingreso y diagnóstico del aneurisma, $14 \%$ se trató dentro de las primeras 48 horas posterior al ingreso, y 14\% recibio tratamiento despues del 3 día de realizado el diagnóstico.

Finalmente a los 30 días del ictus se valoró la evolución de los pacientes utilizando la escala de rankin modificada (mRS), que se describe en la Tabla 5.

Entre los pacientes sin datos y perdidos en la evolución en el trabajo suman el $33 \%$ de los casos, la mitad de estos son pacientes que no respondieron el teléfono o en ese momento había sido cambiado. Estos 12 pacientes podrían corresponder a cualquiera de las categorías de $\mathrm{mRS}$, si bien sabemos que no presentaron muerte precoz dentro de los primeros días.

\section{Discusión}

En Uruguay, según el último censo de 2011, la población total contabilizada fue de 3.876 .314 personas, y el sexo femenino está representado por el $52 \%$ del total de habitantes ${ }^{12}$. Como se menciono anteriormente es un trabajo con datos preliminares, pero en el mismo ya se evidenció que la HSAea predomina en el sexo femenino con gran claridad, siendo casi el doble en frecuencia en comparación al masculino. Por lo tanto, este parece ser un factor de riesgo vinculado a la formación de los aneurismas puesto que son casi 2 veces más probables en mujeres que en hombres. Se sabe que el pico de prevalencia de aneurismas en mujeres es entre los 40 y 69 años, y esto se correlaciona con una incidencia pico de presentación de hemorragia subaracnoidea, lo cual coincide con los resultados encontrados en este trabajo ${ }^{13}$.

Los afrodescendientes son, desde el punto de vista
(\%) de pacientes $(n=36)$

$$
11 \%
$$$$
14 \%
$$

$6 \%$

$3 \%$

$3 \%$

$0 \%$

$30 \%$ cuantitativo, la principal minoría étnico-racial del país; 8,1\% de la población total, y en esta casuística se observó $3 \%$ de pacientes afrodescendientes, lo cual no parecería ser un factor de riesgo como lo es en otros países comparando la literatura internacional ${ }^{14}$. A su vez, podemos reflejar también que a pesar de estar en un contexto social con muchos inmigrantes provenientes de diferentes países de Latinoamérica residiendo en Uruguay, solo dos casos fueron de otro país, y el $94,4 \%$ eran de nacionalidad uruguaya.

De los pacientes analizados, solo 5,6\% tuvo un antecedente personal y/o familiar de aneurisma, lo que puede sugerir un origen esporádico de la enfermedad en la mayoría de los casos $^{15}$.

La hipertensión arterial está asociada en el $64 \%$ de los casos, y el tabaquismo en $50 \%$, como mostraron nuestros resultados. En Uruguay cerca del $40 \%$ de la población tiene hipertensión arterial y el $18 \%$ son fumadores, si comparamos estos valores con respecto a los pacientes con HSAea, observamos que el grupo de los fumadores aumenta a más del doble (de 18 al 50\%) en esta enfermedad. Estos hallazgos sugieren una fuerte vinculación entre el hábito tabáquico con la formación de aneurismas y HSAea ${ }^{16}$.

En numerosas ocasiones el diagnóstico de hemorragia meníngea resulta difícil por diferentes motivos, síntomas inespecíficos o el equipo de salud inexperiente, retrasando el diagnóstico y tratamiento, exponiendo al paciente a complicaciones como el resangrado con alta morbimortalidad. Los resultados expresan $25 \%$ de consultas previas antes del diagnóstico, y en el $5,6 \%$ de estos casos consultaron múltiples veces, siendo dados de alta sin diagnóstico. En esos casos hay un error del equipo de salud en no detectar la enfermedad precozmente. Es más bajo que en el trabajo nacional publicado en el 2016, donde se encontró un error diagnóstico del $31,1 \%$, pero sigue siendo alto con respecto a las publicaciones internacionales de la última década: 5,4\%$14,8 \%{ }^{17}$.

A grandes rasgos la distribución topográfica de los aneurismas encontrados ya sea rotos y no rotos es similar a lo publicado en otros trabajos ${ }^{18}$. Del total de los aneurismas, el comunicante anterior, el silviano y comunicante posterior se encontraron como rotos en el $80 \%$ de los casos. Estos hallazgos podrían sugerir que los aneurismas del sector anterior 
pueden tener más tendencia a romperse.

No tuvimos casos de aneurismas de la circulación posterior, lo cual nos llama la atención ya que comparando con la bibliografía internacional estos representan entre 6 y $22 \%{ }^{19}$. Este resultado puede estar vinculado a que gran parte de los pacientes se realizaron diagnóstico de aneurisma con angio TC, el cual disminuye la sensibilidad para detectar aneurismas del sector posterior ${ }^{20}$.

El tiempo entre realizado el diagnóstico y el tratamiento del aneurisma, es un elemento importante dado que expone al paciente a complicaciones de alta morbimortalidad como es el resangrado ${ }^{21}$. Nuestros resultados muestran que en la gran mayoría de los pacientes que recibieron tratamiento (86\%), el mismo se realizó dentro de las primeras 48 horas, lo cual nos habla de que en Uruguay una vez diagnosticado el aneurisma, la mayoría se trata de manera precoz.

El GCS y sus componentes individuales tienen una correlación con el resultado a largo plazo, siendo de valor predictivo en cuanto al pronóstico. Si analizamos el estado neurológico al momento del diagnóstico y pacientes fallecidos a los 30 días, vemos que el $100 \%$ de los fallecidos presentaron un valor en la escala de la WFNS al ingreso de 4 y 5 . La misma relación ocurre con la escala de $\mathrm{HyH}$ al ingreso y muerte a los 30 días por lo que concluimos que el estado clínico inicial es un factor de gran importancia a tener en cuenta que influye en la evolución y pronostico del paciente ${ }^{22}$.

Los pacientes que recibieron tratamiento del aneurisma son 29 (77\% del total de pacientes), de los cuales el $82 \%$ se realizó clipado quirúrgico, en el $18 \%$ restante embolización con coils. Aproximadamente, la mitad de los paciente para cualquiera de los 2 tipos de tratamiento presentó en la evolución un mRS entre 0 y 2. La proporción de pacientes fallecidos es de $13 \%$ y $40 \%$ para tratamiento quirúrgico y endovascular respectivamente. Debemos aclarar que esos dos pacientes que se embolizaron y fallecieron, tenían una HSA grave desde el inicio, con WFNS 5, lo cual tiene impacto pronóstico como mencionamos anteriormente. De todas maneras habría que realizar un estudio con mayor número de pacientes para tomar una conclusión con real valor estadístico.

No hay diferencia con respecto a la topografía de los aneurismas según el tipo de tratamiento que se realice. Los resultados evidencian que el $60 \%$ de los casos de aneurismas embolizados corresponden a aneurismas silvianos y el $40 \%$ a aneurismas de topografía comunicante anterior. En cuanto a los aneurismas clipados el $26 \%$ fueron silvianos, el $30 \%$ comunicante anterior y el 34\% comunicante posterior. Por lo tanto, podemos decir que la topografía del aneurisma no fue una variable que determinó el tratamiento elegido. Por lo anteriormente analizado podemos decir que en Uruguay el tratamiento de los aneurismas más frecuentemente utilizado es el clipado quirúrgico. Esta realidad no coincide con los resultados internaciones, en donde los tratamientos endovascualres tienen más porcentaje que el observado aquí ${ }^{23}$, lo cual suponemos tiene que ver con que las dos opciones terapéuticas no están incluidas de la misma manera en el sistema integrado de salud de nuestro país. Pensamos que sería de gran utilidad completar el estudio más adelante para poder afirmar esta conclusión.

En cuanto a las complicaciones vinculadas al tratamiento realizado, tuvimos $25 \%$ de complicaciones globalmente, y si las separamos por cada tratamiento, el quirúrgico fue casi el doble $(66 \%)$ del endovascular $34 \%$. Hay que ser cuidadosos al analizar dicha casuística porque es un $n$ bajo, y no podemos sacar conclusiones significativas. De todas maneras cuando lo comparamos con la literatura internacional, nuestros porcentajes de complicaciones son más altos, y en la bibliografía luego de muchos estudios (ISAT, BRAT) se llegó a la conclusión que no hay diferencia significativa en cuanto al porcentaje de complicaciones por una técnica u otra, lo que si se vió fue mayor tasa de curación con el tratamiento quirúrgico $^{24,25}$. Un factor que seguramente incide en los porcentajes mostrados es que los trabajos internacionales se refieren a resultados de un solo Centro, con un neurocirujano senior con amplia experiencia en la cirugía de los aneurismas. En este trabajo se analizan todas las HSA que ingresan en distintos Centros y son tratados por neurocirujanos de guardia, a veces con muy poca experiencia en neurocirugía vascular. Quizás una conclusión sería que debería haber en Uruguay centros de referencia donde enviar los pacientes con HSA. En dichos centros serían tratados por neurocirujanos con dedicación vascular, lo que evidentemente redundaría en una mejoría de los resultados. A diferencia de lo que sucede con la cirugía convencional, la cirugía endovascular se realiza en 3 centros y siempre por los mismos profesionales con amplia dedicación a tratamientos endovasculares.

\section{Conclusión}

Este trabajo presenta los primeras cifras sobre las características epidemiológicas de la HSAea en Uruguay. A pesar de ser datos preliminares de un trabajo global se puede concluir que nuestra población de riesgo al igual que en el resto de la literatura internacional son las pacientes de sexo femenino entre los 40 y 60 años de edad; y, que hay una fuerte asosciación entre el hábito tabáquico y la HSA. A su vez, a diferencia del resto de la bibliográfia aquí se evidenció que nuestro país tiene aún un alto error diagnóstico, así como un porcentaje elevado de pacientes que se realiza tratamiento quirúrgico y no endovascular.

\section{Referencias}

1. Warlow C, Gijn J, Dennis M,Wardlaw J, Bamford J, Hankey G, et al Stroke Practical Management. Tercera Edición, 2008, Cap 9, Pag 457-502.

2. McDougali C, Spetzler R, Zabramski J, Partovi S, Hills N, Nakaji $\mathrm{P}$, et al. The Barrow ruptured Aneurysms Trial. J. Neurosurg 116: 135-144, 2012.

3. Rubin D. Matched Sampling for Causal Effects. Cambridge University Press, 2006.

4. Al-Tamimi $Y$, Bhargava D, Feltbower R, Hall G, Goddars A, Quinn A, et al. Limbar Drainage of Cerebroespinal Fluid After Aneurysmal Subarachnoid Hemorrhage: A Prospective, Randomized, Controlled Trial (LUMAS). Journal of the American Heart Association, American Stroke Association, Enero, 2012.

5. Breiman L. Random Forests. Publication date 2001. Journal name Machine Learning, Volume 45, Issue 1, Pages 5-32. Pu- 
blisher Springer Netherlands.

6. Oliveira J, Beck J, Ulrich C, Rathert J, Raabe A, Seifert V. Comparison between clipping and coiling on the incidence of cerebral vasospasm after aneurysmal subarachnoid hemorrhage: a systematic review and meta-analysis. Neurosurg Rev, 2007; 30:22-31.

7. Quiñones A, Hindosa. Schmidek y Sweet Operative Neurosurgical Techniques. Sexta Edición, volumen 2, 2012, sección vascular.

8. R. Gillum, P. Gorelick, E. Cooper: Stroke in Blacks. A Giude to Management and Prevention. 1999, pag 29-35.

9. Breiman L. Classification and regression trees. Publication data 1984. Publisher Chapman \& Hall/CRC.

10. Quereshi A,Suri A, Nasar A, Kirmani J, Divani A, He W, et al. Trends in Hospitalization and Mortality for Subarachnoid Hemorrhage and Unruptured Aneurysms in the United States. Neurosurgery 57:1-8, 2005.

11. Gonzalez N, Dusick J, Duckwiler G, Tateshima S, Jahan R, Martin $\mathrm{N}$, et al. Endovascualr Coiling of Intracranial Aneurysma in Elderly Patients: Report of 205 Trates Aneurysms. Neurosurgery 66: 714-721, 2010.

12. INE, Censo 2011. Total país. Síntesis de resultados. Disponible en http://www.ine.gub.uy/.

13. Vivancos J, et al. Guía de actuación clínica en la hemorragia subaracnoidea. Sistemática diagnóstica y tratamiento. Neurología. 2012. doi:10.1016/j.nrl.2012.07.009.

14. T. Ishibashi, Y. Murayama, M. Urashima, T. Saguchi, M. Ebara, H. Arakawa, K. Irie, H. Takao, T. Abe: Unruptured Intracranial Aneurysms: Incidence of Rupture and Risk Factors. Journal of the American Heart Association, American Stroke Association, Enero, 2009, pag 313-317.

15. D. Kim, G. Ginhoven, D. Milewicz: Incidence of Familial Intrcranial Aneurysms in 200 Patients: Comparison Among Caucasian,
African-American, and Hipanic Popilations. Neurosurgery 53: 302-308, 2003.

16. Y. Kim, D. Neal, B. Hoh: Risk Factors, Incidence, and Effect of Cardiac Failure and Myocardial Infarction in Aneurysmal Subarachnoid Hemorrhage Patients. Neurosurgery 73:450-457, 2013.

17. V. Benancourt, A. Jaume, C. Aboal. Errores diagnosticos en la Hemorragia Subaracnoidea Aneurismatica. Rev. urug. med. Interna, ISSN: 2393-6797 - Diciembre 2016 N³: 62-68.

18. Rinne JK, Hernesniemi JA. De Novo Aneurysms: Special Multiple Intracranial Aneurysms. Neurosurgery. 1993;33(6):981-5.

19. C. Cognard, A. Dorfler, M. Forsting, W. Kuker, L. Pierot, L. SpeIle, I. Szikora, I. Wanke. Intracranial Vascular Malformations and Aneurysms. 2 edición, 2008.

20. Chen W; Wang J; Xin W. Accuracy of 16-ROW Multislice computed tomographic angiography for assessment of small cerebral aneurysms. Neurosurgery 62:113-122, 2008.

21. P. Lasjaunias, K. Ter. Brugge, A. Berenstein: Surgical Neuroangiography. Segunda edición, 2006, tomo 2.1, 217-333.

22. Guresir E; Beck J; Vatter H. Subarachnoid Hemorrhage and intracerebral hematoma: incidence, prognostic factors, and outcome. Neurosurgery 63: 1088-1094, 2008.

23. Li H, Pan R et al. Clipping Versus Coiling for Ruptured Intracranial Aneurysms: A Systematic Review and meta-analysis. Stroke 2013;44:29-37.

24. Molyneux A., et al. International subarachnoid aneurysm trial (ISAT) of neurosurgical clipping versus endovascular coiling in 2143 patients with ruptured intracranial aneurysms: a randomized comparison of effects on survival, dependency, seizures, rebleeding, subgroups, and aneurysm occlusion. Lancet 2005; 366: 809-17.

25. Spetzler,R et al. The Barrow Ruptured Aneurysms Trial: 6-year results. J. Neurosurg. 26: 1- 9, 2015. 